\title{
FORMATE FISCHER-TROPSCH PROCESS FOR PRODUCING TRADITIONAL ENERGY CARRIERS WITH ZERO CARBON BALANCE
}

\author{
MIKHAIL GOTOVSKY, ALEXANDER GOTOVSKY, VITALY LYCHAKOV, \\ VLADIMIR MIKHAYLOV, YURY SUKHORUKOV \& EKATERINA SUKHORUKOVA \\ Polzunov Institute, Russia
}

\begin{abstract}
The Fischer-Tropsch process (F-T process) is known as a method of synthesizing organic products (including liquid fuel) from $\mathrm{CO}+\mathrm{H}_{2}$ mixture gases (synthesis gas) using various catalysts. The interest in the F-T process is associated with the global struggle against carbon emissions. Earlier we noted that the electrochemical synthesis of formic acid from water and atmospheric $\mathrm{CO}_{2}$ can be a promising general scheme for obtaining "green fuel" with minus (negative) "carbonic effect" simultaneously "formate cycle" (synthesis of organic products from formic acid produced from atmospheric $\mathrm{CO}_{2}$ ) fuel has zero carbon balance. This synthesis is brought to efficiency, surpassing natural photosynthesis. Synthesized formic acid can be used as a fuel for fuel cells and as a hydrogen accumulator. A semiproduct of green fuel synthesis with zero carbon balance and synthetic organic chemical products with minus carbon balance (thanks to bonded carbon from atmospheric $\mathrm{CO}_{2}$ ). From an economical point the direct use of formic acid would be more rational (with the exception of one stage), without first carbon monoxide separation. From the point of view of the physical chemistry, it is necessary to take into account the relatively low reactivity of formic acid in comparison with carbon monoxide. The traditional way of activation, by applying high temperatures, high pressures and specific catalysts in gas phase. However, using electromagnetic activation may be a more interesting pathway. This aspect will be discussed in this work. The final selection of the method based on the results of bench scale should be made on the basis of a complex criterion of the rate of passage of the modified F-T process and economic indicators: equipment cost, and energy consumption in terms of the mass of final products, taking into account of their cost. In this case, it appears an alternative way of obtaining some final products without using fossil hydrocarbons.
\end{abstract}

Keywords: Fischer-Tropsch process, formate cycle, green fuel, electromagnetic activation, zero carbon balance.

\section{INTRODUCTION}

The Fischer-Tropsch process (F-T process) has been known since the 1920s as a method of obtaining a number of organic products from a mixture of $\mathrm{CO}+\mathrm{H}_{2}$ gases produced by treating coal with water vapor. The mixture of $\mathrm{CO}+\mathrm{H}_{2}$ later was referred as synthesis gas. The same mixture can be received by non-oxidative pyrolysis of timber waste.

Depending on the ratio of the synthesis gas components, using different catalysts (usually transition metals from the series: iron, cobalt, nickel and copper), the following redox reactions take place:

$$
\mathrm{CO}+2 \mathrm{H}_{2}->-\mathrm{CH}_{2-}+\mathrm{H}_{2} \mathrm{O}
$$

or

$$
2 \mathrm{CO}+\mathrm{H}_{2}->-\mathrm{CH}_{2}-+\mathrm{CO}_{2} .
$$

The symbol $-\mathrm{CH}_{2}$ - is a mixture of organic products similar to alkane, resulting in each of the two reactions. The ratio of different organics depends on the physical conditions (temperature, pressure), on the catalyst used, and on the residence time in the reaction zone. 
During the Second World War, various versions of the F-T process were used in Germany and Japan to produce synthetic liquid fuels (ersatz gasoline), diene monomers of synthetic rubber (for car tires), as well as aromatics (hereafter processed into paints, varnishes and pharmaceuticals ). In 1944, ersatz gasoline was produced in Germany in the amount of 124,000 barrels per day (6.5 million tons per year). In the post-war period, interest in the F-T process recurred during the 1973 oil crisis, but later disappeared when oil and natural gas prices fell.

In the 1980s, in Brazil, the F-T process was considered as one of the ways to produce synthetic liquid fuels from forest waste, but biotechnological approaches for processing into fuel alcohol (bioethanol) was recognized as more profitable.

Nowadays the interest in the F-T process is motivated by the problem of the global struggle with greenhouse gases, primarily with carbon emission. Carbon emission refers to the release of $\mathrm{CO}_{2}$ from the combustion of hydrocarbon fuels derived from mineral raw materials: oil, natural gas, and coal. Owing to this struggle (the Kyoto Protocol, the Paris Agreement, etc.), the processes of producing "green fuel" with a "zero carbon-carbon balance" have become very relevant. All carbon in such fuels should be obtained by binding atmospheric $\mathrm{CO}_{2}$, and subsequent combustion only returns the same $\mathrm{CO}_{2}$ to the atmosphere. Also becoming relevant are industrial processes with a "minus carbon balance", i.e. those in which synthetic chemical products contain fixed carbon from atmospheric $\mathrm{CO}_{2}$.

In [1], [2], it was noted that a promising general scheme for obtaining green fuel and products with a minus carbonic effect can be the "formate cycle". The basis of the formate cycle is the electrochemical production of formic acid from water and atmospheric $\mathrm{CO}_{2}$ :

$$
\mathrm{CO}_{2}+2 \mathrm{H}_{2} \mathrm{O}+2 \mathrm{e}^{-}->\mathrm{HCOOH}+2 \mathrm{OH} \text {. }
$$

This process has been known since 1869, from the experiments of Nikolai Beketov from his electrochemical synthesis of sodium bicarbonate formates experiments. Much later, in the 2010 s, due to the interest in "green energy", the process was brought (at lab scale) to a level even more energy-efficient than natural photosynthesis (that is, than the process of binding $\mathrm{CO}_{2}$ in the form of carbohydrates to plants).

The resulting formic acid can be used in several ways, in particular:

1. As fuel for fuel cells;

2. As a hydrogen accumulator (hydrogen is released by the reaction $\mathrm{HCOOH}->\mathrm{CO}_{2}+\mathrm{H}_{2}$ );

3. As an intermediate for the synthesis of green fuel with zero (neutral) carbon balance, and synthetic organic chemical products with a minus carbon footprint.

With respect to the third point to catalytically convert formic acid into synthesis gas components necessary for the F-T process:

$$
\begin{aligned}
& \mathrm{HCOOH}->\mathrm{CO}_{2}+\mathrm{H}_{2}, \\
& \mathrm{HCOOH}->\mathrm{H}_{2} \mathrm{O}+\mathrm{CO} .
\end{aligned}
$$

At the same time, the quality of the synthesis gas that should be obtained this way will be much higher than in the original process dating from the 1920s-1940s. There, synthesis gas was obtained from coal or from biological waste, and inevitably contained impurities of sulfur and nitrogen compounds, acting as catalytic poisons and preventing the selective conduct of the process, aimed at a number of pre-defined products.

In the case of the formate cycle, synthesis gas will generally not contain catalytic poisons and other components that reduce the selectivity of the F-T process, which will allow the use of a number of existing developments that require high purity at the inlet. 
The disadvantage of implementing this approach is the need for three separate stages:

1. The release of hydrogen from formic acid;

2. Isolation of carbon monoxide from formic acid;

3. The actual F-T process.

A more rational approach would be the direct use of formic acid, without the preliminary isolation carbon monoxide.

At the level of chemical reactions, such an analogue of the F-T process would look like:

$$
\mathrm{HCOOH}+2 \mathrm{H}_{2}->-\mathrm{CH}_{2-}+2 \mathrm{H}_{2} \mathrm{O} \text {, }
$$

or

$$
2 \mathrm{HCOOH}+\mathrm{H}_{2}->-\mathrm{CH}_{2}-+\mathrm{CO}_{2}+2 \mathrm{H}_{2} \mathrm{O} \text {. }
$$

The closest approach linked to this concept is the catalytic reduction (with $\mathrm{H}_{2}$ ) of formic acid to methanol:

$$
\mathrm{HCOOH}+2 \mathrm{H}_{2}->\mathrm{CH}_{3} \mathrm{OH}+\mathrm{H}_{2} \mathrm{O} .
$$

From a physical chemistry point of view the process under consideration, it is necessary to take into account the relatively low reactivity of formic acid in comparison with carbon monoxide. However there is a traditional way of activating it, which is by applying high temperatures, high pressures in gas phase, by designing specific catalysts.

However, an electromagnetic activation may be more interesting.

The theory of electromagnetic activation today is not sufficiently developed to allow calculating what kind of electromagnetic effect would lead to a satisfactory reaction rate, and which will be optimal from the point of view of energy consumption for the process.

Based on the chain free-radical version or the ion-radical version of the process, activation by an electrostatic field at the field strength near the physical boundary of the breakdown (occurrence of a corona gas discharge) may be promising [3].

Another option could be the exposure to microwave radiation with a wavelength of about $0.1 \mathrm{~mm}$ (i.e. in the overlap region with the infrared range, in which absorption peaks of the carbon-oxygen bond are possible with appropriate excitation of this bond and, accordingly, its activation) [4].

It should also be noted the possibility to combine electromagnetic and catalytic effects on the reaction medium, in particular, the use of the catalytic effect of the micro-needle surface, on which, when an electric potential is applied, many areas with high field strength or multiple corona discharge can be created. Even with a relatively small imposed electrical potential, there are a number of specific physicochemical effects associated with the local electric field strength around the microneedles, as noted in the work of Zaitsev in 2017 [5].

\section{MILLER-UREY EXPERIMENTS}

A significant amount of data on such organic synthesis reactions has been accumulated since 1953, which would be the experiments of Stanley Miller and Harold Urey [6]. These experiments were not planned as technologically oriented, but were carried out to test hypotheses of pre-biological evolution on Earth, which began about 4 billion years ago, in a reducing atmosphere containing hydrogen, methane, ammonia, carbon monoxide, and water vapor. The mixture of gases was exposed to a spark discharge simulating the periodic lightning in the atmosphere. Despite the low temperature and low intensity of electrical discharges, the degree of conversion to higher molecular weight organic compounds ranged from $10-15 \%$ after 170 hours. 
Subsequently, the Miller-Urey experiments were reproduced under different conditions and showed that the yield of various organic compounds depends on the local electric fields at the surface of the solid phase.

Thus, with sufficient confidence it can be said that there is a mixed effect of the classical catalysis and electrical field initiation for a series of chemical reactions including the formation of new carbon-carbon bonds in the region of locally high field strength on a solid inorganic surface. This can serve as a starting point for the development of technological methods of a modified F-T process, based on a direct gas-phase reaction of formic acid with hydrogen, using a combination of inexpensive catalysts and an electric field.

Such methods theoretically open up the possibility not only for the reaction of formic acid with hydrogen, but for reactions with a third component: carbon dioxide. Under normal conditions, carbon in a $\mathrm{CO}_{2}$ molecule exhibits low reactivity, in particular, due to the high symmetry of the molecule and zero dipole moment. However electromagnetic activation can lead to a change in the configuration of the electron orbitals, and the deviation of the molecule from a strictly linear structure. In this case, we can assume in the three-component system the following reactions:

$$
\begin{gathered}
\mathrm{HCOOH}+\mathrm{CO}_{2}->\mathrm{HCOOH}-\mathrm{COOH} \text { (oxalic acid formation), } \\
\mathrm{HOOC}-\mathrm{COOH}+\mathrm{H}_{2}->\mathrm{HOOC}-\mathrm{CHO}+\mathrm{H}_{2} \mathrm{O} \text { (reduction to glyoxylic acid). }
\end{gathered}
$$

Glyoxylic acid is already quite high reactivity. Also, for any of the two types of electromagnetic activation discussed, it is possible to assume with high probability that the reaction proceeds in parallel:

$$
\mathrm{CO}_{2}+\mathrm{H}_{2}->\mathrm{HCOOH} \text {. }
$$

Thus, in the above three-component system, an additional mole of $\mathrm{CO}_{2}$ per mole of $\mathrm{HCOOH}$ can be involved in the F-T process, and an additional amount of hydrogen can be involved in binding $\mathrm{CO}_{2}$, which greatly increases the efficiency of the proposed process for utilizing atmospheric carbon dioxide.

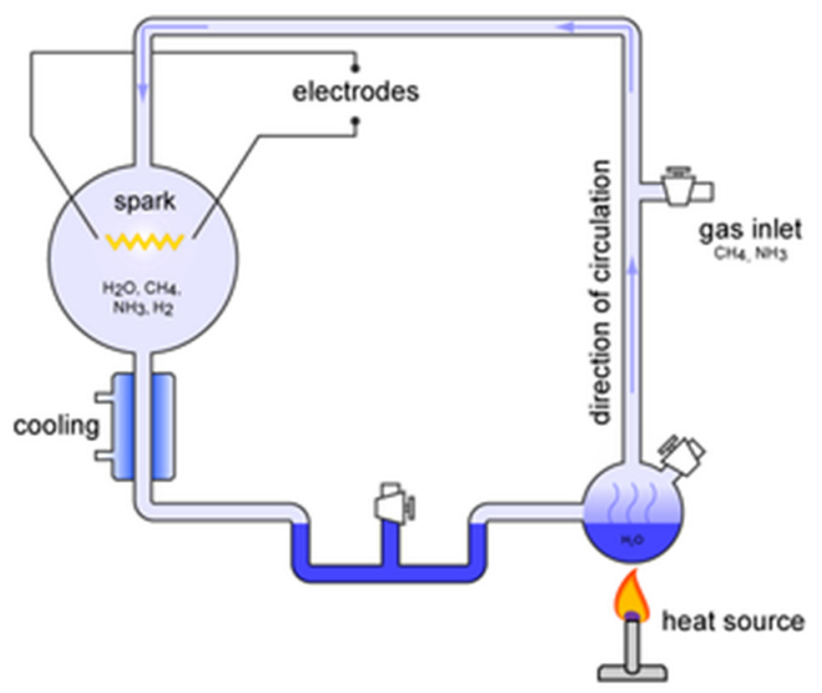

Figure 1: Scheme of Miller-Urey experiment. 


\section{USING DISPROPORTION (TRANSMUTATION) OF FORMIC ACID}

Disproportionation, sometimes called dismutation, is a redox reaction in which a compound of intermediate oxidation state converts to two different compounds, one of higher and one of lower oxidation states. Although not widely accepted, disproportionation is sometimes used to describe any desymmetrizing reaction of the following type: $2 \mathrm{~A} \rightarrow \mathrm{A}^{\prime}+\mathrm{A}^{\prime \prime}$, regardless of any redox process. In other words disproportionation is a chemical reaction, typically a redox reaction, where a molecule is transformed into two or more dissimilar products. In a redox reaction, the species is simultaneously oxidized and reduced to form at least two different products.

Such exception would also be linked to the second preliminary stage which is the release of hydrogen from formic acid. Such an optimal analogue of the F-T process would involve a redox disproportionation of formic acid (dismutation and asymmetrization of the initial substance by hydrogen).

At of chemical reaction level, such a process could be presented such as:

$$
3 \mathrm{HCOOH}->-\mathrm{CH}_{2-}+2 \mathrm{CO}_{2}+2 \mathrm{H}_{2} \mathrm{O} \text {. }
$$

The most comparative process applied at lab scale is the disproportionation (dismutation) of benzoic acid or its salts in the presence of a catalyst:

$$
\mathrm{C}_{6} \mathrm{H}_{5} \mathrm{COOH}->\mathrm{C}_{6} \mathrm{H}_{6}+\mathrm{C}_{6} \mathrm{H}_{4}(\mathrm{COOH})_{2} \text {. }
$$

It is also known reduction-oxidation disproportionation of carbon monoxide:

$$
2 \mathrm{CO}->\mathrm{C}+\mathrm{CO}_{2},
$$

and reduction-oxidation disproportionation of formaldehyde:

$$
2 \mathrm{HCHO}+\mathrm{H}_{2} \mathrm{O}->\mathrm{CH}_{3} \mathrm{OH}+\mathrm{HCOOH} \text {. }
$$

It should be noted that the second of the known paths leading to the Fischer-Tropsch reaction goes as follows:

$$
2 \mathrm{CO}+\mathrm{H}_{2}->-\mathrm{CH}_{2-}+\mathrm{CO}_{2} \text {. }
$$

The latter partly involves also disproportionation.

Also partly disproportionation will be the reaction of formic acid and hydrogen:

$$
2 \mathrm{HCOOH}+\mathrm{H}_{2}->-\mathrm{CH}_{2-}+\mathrm{CO}_{2}+2 \mathrm{H}_{2} \mathrm{O} \text {. }
$$

Exploring the possible ways to disproportionate formic acid (or formates) is a nontrivial task, but the result looks very promising in an economic sense in two obvious ways. First way is the decrease of number of process stages. Second way is absence of costly catalysts. Both ways are typical for cost reduction in chemical technology. The one-step production of synthetic green fuel from formic acid, which is almost identical to gasoline, will allow, without additional changes, to transfer classic motor transport to a fuel scheme with zero carbon balance. This will minimize the costs of implementing measures related to the Kyoto Protocol and the Paris Agreement.

\section{PROPOSALS FOR DEVELOPMENT ELECTROMAGNETIC ACTIVATION}

The practical steps to develop a new formate based F-T process can be performed at a laboratory scale to identify possible reaction paths. In particular, it can be achieved by verification of versions of effective disproportionation in a strong electric and/or electromagnetic field. 
In this regard, there is an analogy with the above-mentioned possibilities of electromagnetic initiation of a series of reactions of formic acid with hydrogen, as well as with carbon dioxide, but with a wider variety of possible methods. Overall, three broad classes of methods of activation of the electric (electromagnetic) field could be considered:

1. Activation in a homogeneous gaseous environment: this involves an activation in the corona gas discharge of an electrostatic field, or an activation by microwave radiation with a wavelength of about $0.1 \mathrm{~mm}$ (mentioned above);

2. Activation in a two-phase medium with two continuous phases or with a dispersed liquid phase: this is for example an activation by a glow discharge in the gas phase in contact with the liquid phase (in particular, with aerosol droplets);

3. Activation in a non-stationary two-phase medium with a dispersed gas phase: this is the activation method in a fluid when applying a high power ultrasonic field. When this occurs, a phenomenon of cavitation with luminescence effects upon the collapse of microbubbles (sonoluminescence).

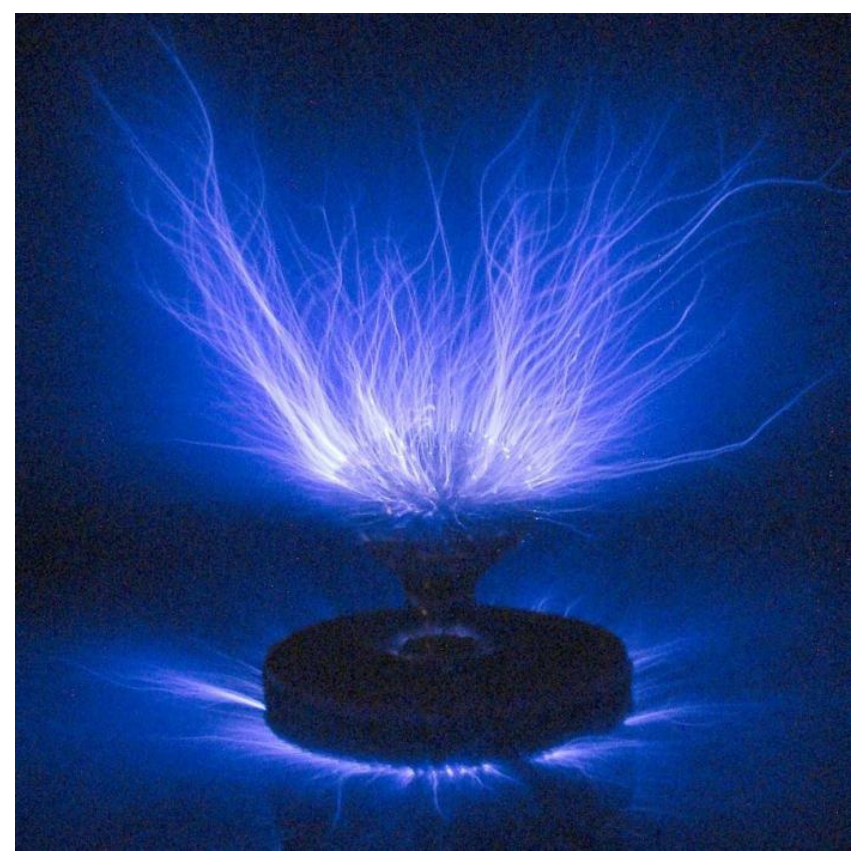

Figure 2: Corona discharge photography.

The mechanism of sonoluminescence is still insufficiently studied, but the excitation of interatomic bonds is undoubted, since only these effects can explain the high-brightness light flashes [7].

"Sonochemistry is studied primarily by chemists and sonoluminescence mainly by physicists, but a single physical phenomenon - acoustic cavitation - unites the two areas. The physics of cavitation bubble collapse is relatively well understood by acoustical physicists but remains practically unknown to chemists. By contrast, the chemistry that gives rise to electromagnetic emissions and the acceleration of chemical reactions is familiar to chemists, but practically unknown to acoustical physicists" [8]. 

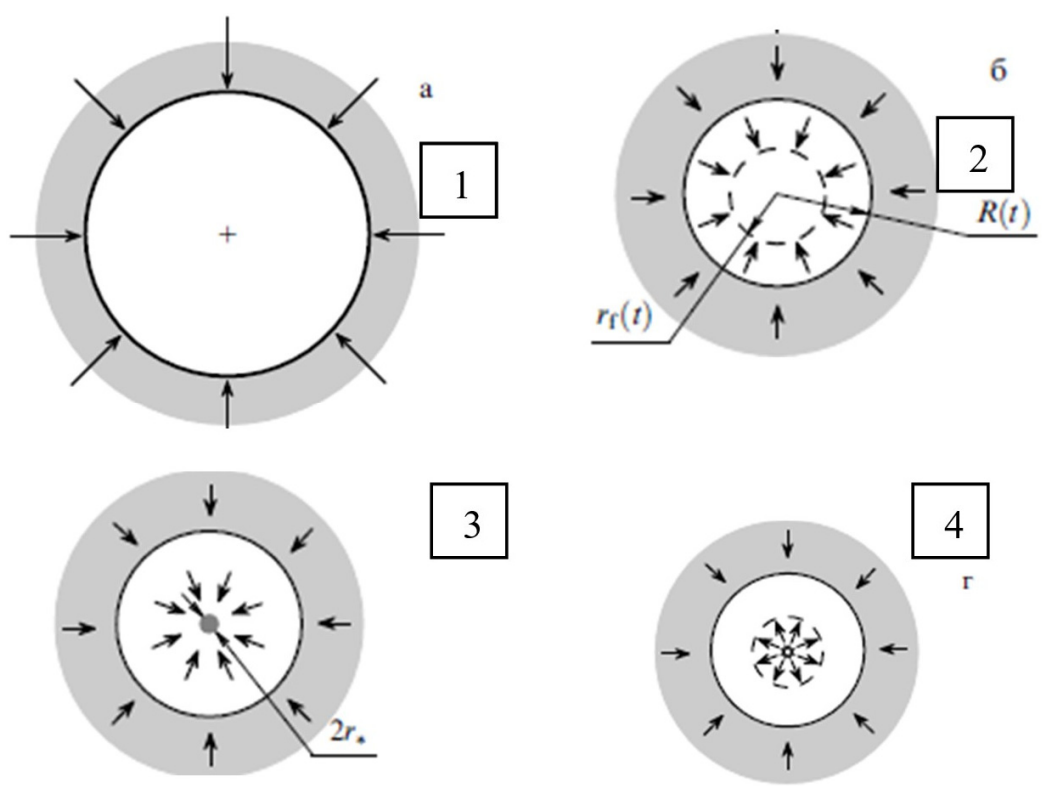

Figure 3: Scheme of sonoluminescence [9]. 1: Compression start; 2: Shock wave formation; 3: Extremal confined plasma; 4: Expansion of the compression zone. $\mathrm{R}, \mathrm{r}=$ bubble and shock wave radii.

Here it will be useful to mention the great contribution to the study of this process, made by Nigmatulin et al. [9]. Although the main goal of their research was to prove the possibility of the emergence of local thermonuclear fusion under these conditions, they succeeded in significantly increasing the level of description of the physics of sonoluminescence processes.

\section{CONCLUSIONS}

Initiation of the modified F-T process in electromagnetic field allows to organize more technological regimes of producing final products (hydrocarbons) from formic acid bypassing intermediate stages of classic F-T process. In particular high technological value of class 2 and class 3 processes should be noted. And problem of hydrocarbons separation from formic acid and water is easily solved because of hydrocarbons insolubility.

As mentioned above, the final choice of the method based on the results of bench studies should be made on the basis of a complex criterion of the rate of passage of the modified F-T process, the degree of conversion of the starting materials, and economic indicators. The latter involves (but are not limited to) equipment cost, and energy consumption in terms of the mass of the final products, taking into account their cost. In this case, an alternative way of obtaining such end products without using fossil hydrocarbons can play a special role.

Installations facilities with the modified F-T process may be of particular economic importance for regions that don't possess their own sources of hydrocarbons, but that have an excess of electricity produced (due to the presence of nuclear, solar, wind, geothermal, hydropower, or tidal power plants). In such a region, the installation of modified F-T process can, due to atmospheric carbon dioxide, solve the problem of providing its own raw materials for the organic chemical industry, with its own authentic gasoline or diesel (but with zero 
carbon balance), as well as fuel for transport on formate or hydrogen fuel cells. In the case of a liquid-phase class of a modified F-T process, the installation can be sufficiently environmentally friendly to be placed on sites without exclusion zone from natural, agricultural, or residential facilities, which is significant for regions with high population density.

\section{REFERENCES}

[1] Gotovsky, M., Gotovsky, A., Mikhailov, V., Kolpakov, C., Lychakov, V. \& Sukhorukov, Y.,' Formic acid cycle as partial alternative to Allam cycle less expensive and more simple. TI-IJES, 61+1(2), p. 49, 2018,

[2] Gotovsky, M., Gotovsky, A., Mikhailov, V., Lychakov, V., Sukhorukov, Y. \& Sukhorukova, E., Formate cycle: The third way in green energy. ICEBE 2019, Singapore, p. 29, 2019.

[3] Alexankin, M., Monomolecular reactions of organic radical cations in a strong electric field, Doctoral thesis, Kiev, 1984.

[4] Mikheev, A. \& Pankrushina, N. Peculiarities and possibilities of microwave chemistry. Science in Syberia, 21, p. 9, 2010. (In Russian.)

[5] Zaitsev, S., Microostria, Application, Manufacturing, 2017. (In Russian.)

[6] Bada, J., New insights into prebiotic chemistry from Stanley Miller's spark discharge experiments. Chemical Society Reviews, 42(5), pp. 2186-2196, 2013.

[7] Margulis, M.A., Sonochemistry and Cavitation, Langhorne, 1995.

[8] Apfel, R. et al. (eds), Sonochemistry and Sonoluminescence, 1999.

[9] Nigmatulin, R. et al., On thermonuclear processes in cavitation bubbles. Uspekhi Fizicheskikh Nauk, 184(9), pp. 947-960, 2014. (In Russian.) 